\begin{tabular}{|c|l|}
\hline Title & $\begin{array}{l}\text { Structural A nalysis of Polyelectrolyte Film A bsorbing Metal Ion by SA X S Utilizing with X-ray A nomal ous Dispersion } \\
\text { Effect }\end{array}$ \\
\hline Author(s) & $\begin{array}{l}\text { Sugiyama, Masaaki; Mitsui, Takao; Sato, Takashi; A kai, Y oshinori; Soejima, Yuji; Orihara, Hiroshi; Na, Y ang-Ho; } \\
\text { Itoh, Keiji; Mori, Kazuhiro; Fukunaga, Toshiharu }\end{array}$ \\
\hline Citation & $\begin{array}{l}\text { The Journal of Physical Chemistry B, 111(29), 8663-8667 } \\
\text { https://doi.org/10.1021/p0716076 }\end{array}$ \\
\hline Issue Date & 2007-07 \\
\hline Doc URL & http://hdl.handle.net/2115/50776 \\
\hline Type & article \\
\hline File Information & J. Phys. Chem. B 2007, 111.pdf \\
\hline
\end{tabular}

Instructions for use 


\title{
Structural Analysis of Polyelectrolyte Film Absorbing Metal Ion by SAXS Utilizing with X-ray Anomalous Dispersion Effect ${ }^{\dagger}$
}

\author{
Masaaki Sugiyama,*»" Takao Mitsui, ${ }^{\S}$ Takashi Sato, ${ }^{\S}$ Yoshinori Akai, ${ }^{\S}$ Yuji Soejima, ${ }^{\perp}$ \\ Hiroshi Orihara," Yang-Ho Na," Keiji Itoh, ${ }^{\ddagger}$ Kazuhiro Mori, ${ }^{\ddagger}$ and Toshiharu Fukunaga \\ Research Reactor Institute, Kyoto University, Osaka 590-0494, Japan, Graduate School of Engineering, \\ Kyoto University, Kyoto 606-8501, Japan, Research and Development Center for Higher Education, \\ Kyushu University, Fukuoka 812-8581, Japan, and Department of Applied Science and Engineering, \\ Hokkaido University, Sappro 060-8628, Japan
}

Received: February 27, 2007; In Final Form: April 13, 2007

A distribution of $\mathrm{Cu}$ ions in polyelectrolyte film (Nafion) is directly observed with a small-angle X-ray scattering (SAXS) method utilizing an X-ray anomalous dispersion effect. A partial structure factor of the $\mathrm{Cu}$ ions, $G_{\mathrm{AA}}(q)$, can be derived from the SAXS profiles obtained by scanning the incident X-ray energy around the $\mathrm{Cu} \mathrm{K}$ absorption edge. $G_{\mathrm{AA}}(q)$ has two peaks, indicating that the $\mathrm{Cu}$ ions hierarchically distribute in Nafion film. In addition, a standard SAXS also shows that Nafion film has a hierarchical structure. These results mean that the $\mathrm{Cu}$ ions locate in the domain where the hydrophilic bases aggregate.

\section{Introduction}

In recent years, as raising awareness for global warming, low emission energy generation devices have gotten a lot of attention. One of the most expected devices is polymer electrolyte fuel cell (PEFC). PEFC is being used for a power source of electric devices, electric mobiles, and so on. PEFC mainly consists of three parts: an anode, a cathode, and several sheets of polymer electrolyte membrane. ${ }^{1}$ The roles of the membrane are a separator between the electrodes and a conductor of protons.

Nafion film is used for the membrane of PEFC because it has superior resistance to corrosion and thermodynamical stability. As shown in Figure 1, Nafion consists of main chains (polytetrafluorocarbon, PTFE) and graft chains with a sulfate group at the end. Because of this chemical structure, it has been supposed that Nafion film could make micro phase separation in nanoscale: the domains of graft chains with nano size distribute in the PTFE matrix. ${ }^{2-6}$ In addition, it has been also supposed that a proton could propagate in the domains of the graft chains, which include many ionic groups. In other words, the connection of the domain of graft chains could create the pathway for electric conductivity. ${ }^{7}$ This structure model means that the nanostructure is greatly related to the electrostatic features of Nafion film. For example, if the pathway is blocked off for any reason, the electric conductivity of Nafion film deteriorates.

There are still some problems in PEFC for widely practical use. One of the problems is that PEFC starts to lose an electrical output in a long time operation. There are several reasons for the deterioration of the electrical output, such as catalyst degradation at the electrodes, degradation of electric conductivity

\footnotetext{
Part of the special issue "International Symposium on Polyelectrolytes (2006)".

* To whom correspondence should be addressed. Fax:+81-724-51-2635. E-mail: sugiyama@ rri.kyoto-u.ac.jp.

$\doteqdot$ Research Reactor Institute, Kyoto University.

$\S$ Graduate School of Engineering, Kyoto University.

${ }^{\perp}$ Kyushu University.

"Hokkaido University.
}

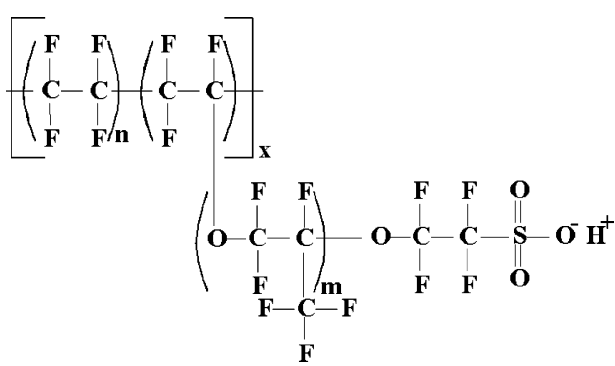

Figure 1. Chemical structure of Nafion.

of the membrane (Nafion film), and so on. Concerning the degradation of electric conductivity, it is deduced that Nafion film could absorb metal ions from the environment, and then the accumulated metal ions make its electric conductivity decrease by blocking the electric pathway as described before. Therefore, it is very important to elucidate the relation between the structure of pathway and the distribution of the absorbed metal ions. In addition, it is expected that the elucidation reveals the mechanism of the degradation of the electric conductivity and also leads to improvement of the electrostatic property of the Nafion film for PEFC.

Small-angle X-ray scattering (SAXS) is one of the most powerful tools to investigate a nanoscale structure. SAXS measurement gives us information of a spatial fluctuation of electron density. From the information, a nanostructure model of Nafion film can be built up by taking into account the difference between the electron density of ion-rich domains and that of PTFE matrix. However, because of the low spatial resolution, the standard SAXS cannot reveal the origin of the fluctuation of the electron density: the fluctuation of the atomic densities or that by heterogeneous distribution of constituent atoms. In other words, the standard SAXS can distinguish high or low electric density domains but cannot tell if many atoms or heavier atoms aggregate in the high electric density domain. It means that we cannot know the distribution of a particular atom only by standard SAXS; we have to clarify the distribution of absorbed metal ions in Nafion film. 
Recently, utilization of an X-ray anomalous dispersion effect was proposed as one answer for clarification of the distribution of a particular atom. Up to now, the X-ray anomalous dispersion effect has been usually utilized to solve a phase problem in an $\mathrm{X}$-ray crystal structure analysis for a large molecule, such as a protein: the method is called "MAD" (multiwavelength anomalous diffraction). ${ }^{8,9}$ Because an atomic form factor of a marked atom drastically changed around its absorption edge, it should be observed that the SAXS intensity coming from the marked atom could also change by scanning the energy of the incident $\mathrm{X}$-ray energy. Following this idea, Fuoss et al. applied the X-ray anomalous dispersion effect in SAXS method (ASAXS) to reveal the distributions of anomalous atoms in a metal alloy. ${ }^{10}$ Moreover, Ballauff et al. also applied the ASAXS method to a colloid particle. ${ }^{11-13}$ On the other hand, we have made an attempt to utilize the ASAXS method to reveal the distributions of anomalous atoms in a gel and a liquid crystal. ${ }^{14,15}$

In this paper, we examine the ASAXS method as the technique to clarify the distribution of particular atom, the absorbed metal ions, in Nafion film.

\section{Theory}

SAXS intensity of a sample with an anomalous atom is given around the absorption edge as follows,

$$
\left.I(\boldsymbol{q}, E)=\left|\int_{v}\right| \rho_{\mathrm{N}}(\boldsymbol{r})+\rho_{\mathrm{A}}(\boldsymbol{r}, E)\right\}\left.\exp (\text { iq } \boldsymbol{r}) \mathrm{d}^{3} \boldsymbol{r}\right|^{2}
$$

where, $\rho_{\mathrm{N}}(\boldsymbol{r})$ and $a_{\mathrm{A}}(\boldsymbol{r})$ are scattering powers of normal atoms and an anomalous one, respectively. Here, to separate the energy-dependent term from the independent terms, $\rho_{\mathrm{N}}(\boldsymbol{r})$ and $\rho_{\mathrm{A}}(\boldsymbol{r}, E)$ are rewritten as follows,

$$
\begin{gathered}
\rho_{\mathrm{N}}(\boldsymbol{r})=f_{\mathrm{N}} a_{\mathrm{N}}(\boldsymbol{r}) \\
\rho_{\mathrm{A}}(\boldsymbol{r}, E)=f_{\mathrm{A}}(E) a_{\mathrm{A}}(\boldsymbol{r})
\end{gathered}
$$

where $a_{\mathrm{N}}(\boldsymbol{r})$ and $a_{\mathrm{A}}(\boldsymbol{r})$ are atomic density distribution functions of normal atoms and an anomalous one, respectively, and $f_{\mathrm{N}}$ and $f_{\mathrm{A}}(E)$ are their atomic form factors. The energy-dependent term, $f_{\mathrm{A}}(E)$, is expressed by

$$
f_{\mathrm{A}}(E)=f_{0 \mathrm{~A}}+f_{\mathrm{A}}^{\prime}(E)+\imath f_{\mathrm{A}}^{\prime \prime}(E)
$$

where $f_{\mathrm{A}}^{\prime}(E)$ and $f_{\mathrm{A}}^{\prime \prime}(E)$ are anomalous dispersion terms that drastically change around the absorption edge. ${ }^{14,15}$ Here, we introduce atomic correlation functions for normal and anomalous atom density distributions.

$$
\begin{aligned}
& g_{\mathrm{NN}}(\boldsymbol{R})=\int_{v} a_{\mathrm{N}}(\boldsymbol{r}) a_{\mathrm{N}}(\boldsymbol{r}+\boldsymbol{R}) \mathrm{d}^{3} \boldsymbol{r} \\
& g_{\mathrm{NA}}(\boldsymbol{R})=\int_{v} a_{\mathrm{N}}(\boldsymbol{r}) a_{\mathrm{A}}(\boldsymbol{r}+\boldsymbol{R}) \mathrm{d}^{3} \boldsymbol{r} \\
& g_{\mathrm{AN}}(\boldsymbol{R})=\int_{v} a_{\mathrm{A}}(\boldsymbol{r}) a_{\mathrm{N}}(\boldsymbol{r}+\boldsymbol{R}) \mathrm{d}^{3} \boldsymbol{r}=g_{\mathrm{NA}}(-\boldsymbol{R}) \\
& g_{\mathrm{AA}}(\boldsymbol{R})=\int_{v} a_{\mathrm{A}}(\boldsymbol{r}) a_{\mathrm{A}}(\boldsymbol{r}+\boldsymbol{R}) \mathrm{d}^{3} \boldsymbol{r}
\end{aligned}
$$

Their Fourier transforms are expressed by

$$
\begin{aligned}
& G_{\mathrm{NN}}(\boldsymbol{q})=\int_{v} g_{\mathrm{NN}}(\boldsymbol{R}) \exp (i \boldsymbol{q} \boldsymbol{R}) \mathrm{d}^{3} \boldsymbol{R} \\
& G_{\mathrm{NA}}(\boldsymbol{q})=\int_{v} g_{\mathrm{NA}}(\boldsymbol{R}) \exp (i \boldsymbol{q} \boldsymbol{R}) \mathrm{d}^{3} \boldsymbol{R}
\end{aligned}
$$

$$
\begin{aligned}
& G_{\mathrm{AN}}(\boldsymbol{q})=\int_{v} g_{\mathrm{AN}}(\boldsymbol{R}) \exp (i \boldsymbol{q} \boldsymbol{R}) \mathrm{d}^{3} \boldsymbol{R} \\
& G_{\mathrm{AA}}(\boldsymbol{q})=\int_{v} g_{\mathrm{AA}}(\boldsymbol{R}) \exp (i \boldsymbol{q} \boldsymbol{R}) \mathrm{d}^{3} \boldsymbol{R}
\end{aligned}
$$

SAXS intensity is expressed by substituting eqs $2-12$ into eq 1 .

$$
\begin{array}{r}
I(\boldsymbol{q}, E)=f_{\mathrm{N}}^{2} G_{\mathrm{NN}}(\boldsymbol{q})+\left|f_{\mathrm{A}}(E)\right|^{2} G_{\mathrm{AA}}(\boldsymbol{q})+ \\
f_{\mathrm{N}} f_{\mathrm{A}}(E) G_{\mathrm{NA}}(\boldsymbol{q})+ \\
f_{\mathrm{N}} f_{\mathrm{A}}(E) G_{\mathrm{AN}}(\boldsymbol{q})(13)
\end{array}
$$

Here, we assume that the atomic density fluctuation could be isotropic in our samples. Under this assumption, the average correlation functions do not have angular dependence as follows,

$$
\begin{aligned}
& \bar{g}_{\mathrm{NN}}(R)=g_{\mathrm{NN}}(\boldsymbol{R}) \\
& \bar{g}_{\mathrm{NA}}(R)=g_{\mathrm{AN}}(\boldsymbol{R})=g_{\mathrm{NA}}(\boldsymbol{R})=g_{\mathrm{AN}}(-\boldsymbol{R}) \\
& \bar{g}_{\mathrm{AA}}(R)=g_{\mathrm{AA}}(\boldsymbol{R})
\end{aligned}
$$

and their Fourier transforms become

$$
\begin{aligned}
& G_{\mathrm{NN}}(q)=\int_{v} 4 \pi R^{2} \bar{g}_{\mathrm{NN}}(R) \frac{\sin (q R)}{q R} \mathrm{~d} R \\
& G_{\mathrm{NA}}(q)=G_{\mathrm{AN}}(q)=\int_{v} 4 \pi R^{2} \bar{g}_{\mathrm{NA}}(R) \frac{\sin (q R)}{q R} \mathrm{~d} R \\
& G_{\mathrm{AA}}(q)=\int_{v} 4 \pi R^{2} \bar{g}_{\mathrm{AA}}(R) \frac{\sin (q R)}{q R} \mathrm{~d} R
\end{aligned}
$$

By substituting eqs 4 and 17-19 into eq 13, SAXS intensity is expressed by

$$
\begin{aligned}
I(q, E)=f_{\mathrm{N}}^{2} G_{\mathrm{NN}}(q)+ & \left|f_{\mathrm{A}}(E)\right|^{2} G_{\mathrm{AA}}(q)+ \\
& 2 f_{\mathrm{N}}\left(f_{0 \mathrm{~A}}+f_{\mathrm{A}}^{\prime}(E)\right) G_{\mathrm{NA}}(q)
\end{aligned}
$$

We are interested in a partial structure factor, $G_{\mathrm{AA}}(q)$ : distribution of an anomalous atom, which should be a metal ion absorbed in Nafion film. In order to derive $G_{\mathrm{AA}}(q)$ from an experimental $I(q, E)$, SAXS measurement should be carried out at three energies around the absorption edge: $E_{1}, E_{2}$, and $E_{3}$. We can eliminate $G_{\mathrm{NN}}(q)$ by making the difference between SAXS intensities observed at two different energies.

$$
\begin{aligned}
\begin{aligned}
\Delta I\left(q, E_{2}, E_{1}\right) & =I\left(q, E_{2}\right)-I\left(q, E_{1}\right) \\
& =\Delta f_{\mathrm{A}}{ }^{2}\left(E_{2}, E_{1}\right) G_{\mathrm{AA}}(q)+ \\
& 2 f_{\mathrm{N}} \Delta f_{\mathrm{A}}^{\prime}\left(E_{2}, E_{1}\right) G_{\mathrm{NA}}(q)
\end{aligned} \\
\begin{aligned}
\Delta I\left(q, E_{3}, E_{1}\right) & =I\left(q, E_{3}\right)-I\left(q, E_{1}\right) \\
& =\Delta f_{\mathrm{A}}{ }^{2}\left(E_{3}, E_{1}\right) G_{\mathrm{AA}}(q)+ \\
& 2 f_{\mathrm{N}} \Delta f_{\mathrm{A}}^{\prime}\left(E_{3}, E_{1}\right) G_{\mathrm{NA}}(q)
\end{aligned}
\end{aligned}
$$

where $\Delta f_{\mathrm{A}}^{\prime}\left(E_{m, E n}\right)=f_{\mathrm{A}}^{\prime}\left(E_{m}\right)-f_{\mathrm{A}}^{\prime}\left(E_{n}\right)$ and $\Delta f_{\mathrm{A}}{ }^{2}\left(E_{m}, E_{n}\right)=$ $\left|f_{\mathrm{A}}\left(E_{m}\right)\right|^{2}-\left|f_{\mathrm{A}}\left(E_{n}\right)\right|^{2}$. With eqs 21 and $22, G_{\mathrm{NA}}(q)$ could be 
TABLE 1: Characteristics of Samples

\begin{tabular}{clccc}
\hline code & \multicolumn{1}{c}{ solution } & $R$ & $\sigma_{1}[\mathrm{~S} / \mathrm{cm}]$ & $\sigma_{2}[\mathrm{~S} / \mathrm{cm}]$ \\
\hline A & & & $<10^{-5}$ & $<10^{-5}$ \\
B & deionized water & 0.159 & 0.0511 & 0.2977 \\
C & $0.1 \mathrm{M} \mathrm{CuCl}_{2}$ & 0.254 & 0.0033 & 0.0075
\end{tabular}

eliminated, and $G_{\mathrm{AA}}(q)$ is given as follows:

$$
\begin{gathered}
G_{\mathrm{AA}}(q)=\left\{\frac{\Delta I\left(q, E_{2}, E_{1}\right)}{\Delta f_{\mathrm{A}}^{\prime}\left(E_{2}, E_{1}\right)}-\frac{\Delta I\left(q, E_{3}, E_{1}\right)}{\Delta f_{\mathrm{A}}^{\prime}\left(E_{3}, E_{1}\right)}\right\} \cdot\left\{\frac{\Delta f_{\mathrm{A}}{ }^{2}\left(E_{2}, E_{1}\right)}{\Delta f_{\mathrm{A}}^{\prime}\left(E_{2}, E_{1}\right)}-\right. \\
\left.\frac{\Delta f_{\mathrm{A}}^{2}\left(E_{3}, E_{1}\right)}{\Delta f_{\mathrm{A}}^{\prime}\left(E_{3}, E_{1}\right)}\right\}^{-1}
\end{gathered}
$$

With experimental data, $I\left(q, E_{1}\right), I\left(q, E_{2}\right)$ and $I\left(q, E_{3}\right)$, and eq 23, we can obtain the partial structure factor of the anomalous atom, $G_{\mathrm{AA}}(q)$.

\section{Experiment}

3.1. Sample. Nafion 117 film, purchased from DuPont, was used. Structural parameters of Nafion 117 are $x=m=1$ and $n$ $=6.5$ as shown in Figure $1(\mathrm{EW}=1100 \mathrm{~g}$ /equiv), and its thickness is $175 \mu \mathrm{m}$ in a dry state. At first, Nafion film was washed with $1 \mathrm{~N} \mathrm{HCl}$ and then rinsed with deionized water to remove residual metal ions. Next, the Nafion film was dried at $80{ }^{\circ} \mathrm{C}$ for $30 \mathrm{~min}$ to remove the water. The above-mentioned procedure was named "initialization": the structure of the initialized sample (sample A) was measured with a standard SAXS method. One sheet of the initialized Nafion film was soaked in deionized water (sample B) at $25^{\circ} \mathrm{C}$ for 2 weeks, which is the usual state as a membrane of PEFC. The other sheet was soaked in $0.1 \mathrm{M} \mathrm{CuCl}_{2}$ solution at $25^{\circ} \mathrm{C}$ for 2 weeks (sample $\mathrm{C}$, expecting that sample $\mathrm{C}$ absorbed not only water but also $\mathrm{Cu}$ ions from the solution. Therefore, we were interested in the $\mathrm{Cu}$ ion distribution in sample $\mathrm{C}$. All samples are sealed into cells with kapton windows.

The weight of Nafion film was measured before every experiment. The increase in the weight ratio was defined by

$$
R=\frac{M_{\mathrm{s}}-M_{\mathrm{i}}}{M_{\mathrm{s}}}
$$

where $M_{\mathrm{S}}$ is the weight of Nafion film after soaking in deionized water or $\mathrm{CuCl}_{2}$ solution and $M_{\mathrm{i}}$ is the weight of initialized Nafion film. In addition, the electric conductivity of the Nafion film was also measured using an AC four-terminal method (Impedance meter: HIOKI 3532-80). Table 1 describes the characteristics of the samples.

3.2 Small-angle X-ray Scattering. A synchrotron light (SL) is a suitable X-ray source for our SAXS experiment with energy sweep because a SL provides intensive X-rays in a wide wavelength range. The used SAXS apparatus was SAXESinstalled at BL10C of the Photon Factory in hte Institute of Materials Structure Science (IMSS), High-Energy Accelerator Research Organization (KEK), Tsukuba, Japan. The energy of the incident X-ray was tuned with a double Si monochromator with a resolution $\Delta E / E \simeq 10^{-4}$, and the scattered X-ray was detected with a one-dimensional detector. The magnitude of the observed scattering vector ranged from $2.5 \times 10^{-2}$ to $2.8 \times$ $10^{-1} \AA^{-1}$.

SAXS intensities were measured at three energies (8970, 8980 , and $8990 \mathrm{eV}$ ) around theCu $\mathrm{K}$ absorption edge (8980 $\mathrm{eV}$ ) and at the Ni K absorption (8331 eV). Since it was not expected that the anomalous dispersion effect of $\mathrm{Cu}$ in the

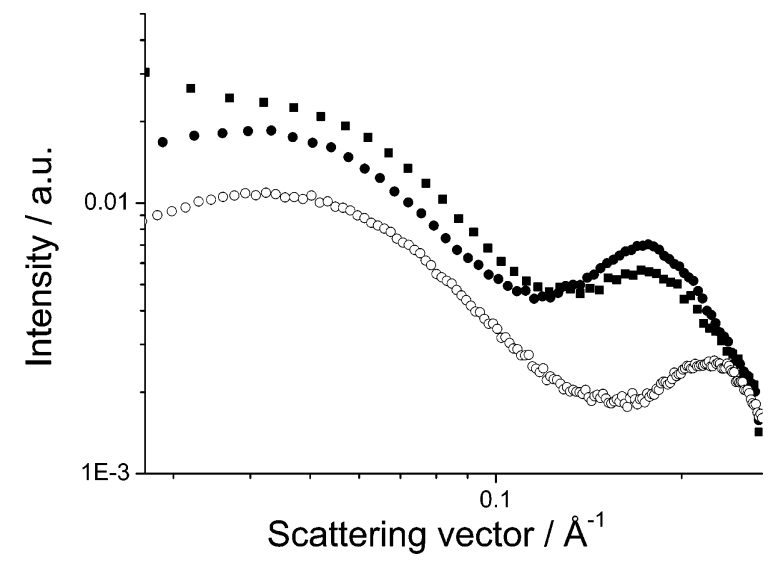

Figure 2. SAXS profiles of samples without any anomalous dispersion effect. Open circles, closed circles, and closed squares denote the SAXS profiles of samples A, B, and C, respectively.

sample could be a major component in the SAXS intensity, the scattered X-ray should be accumulated for a relatively long time, $3600 \mathrm{~s}$, to observe the change in the SAXS intensity around the absorption edge. The observed SAXS intensity was normalized for the amount of the incident X-ray and corrected for the cell scattering and absorption by the kapton window taking in account the transmission.

\section{Results and Discussion}

4.1. Standard SAXS. Figure 2 shows standard SAXS profiles of samples A, B, and $\mathrm{C}$ at $8331 \mathrm{eV}$ (Ni K absorption edge), where no anomalous dispersion effect is expected. As a beginning, we will examine the structure of Nafion film. Two peaks were observed in all the SAXS profiles. This result means that Nafion film does not have a simple phase-separated structure. Comparing the SAXS profiles of samples soaked in solution (samples B and C) with those of the initialized sample (sample A), the second peak position was shifted to $q \simeq 0.18$ $\AA^{-1}$ (samples $\mathrm{B}$ and $\mathrm{C}$ ) from $q \simeq 0.22 \AA^{-1}$ (sample A). In addition, it was also observed in another experiment that the second peak position was shifted to a smaller $q$-position with an increase in the amount of water. ${ }^{18}$ Therefore, the second peak corresponds to the phase-separated structure between the aggragates of the hydrophobic block and those of the hydrophilic block in which the absorbed water is located because the scale of the phase separation is enlarged by the absorption of water. On the other hand, the first peak position $\left(q \simeq 0.05 \AA^{-1}\right)$ did not move as a result of the absorption of water. Considering these results, we propose that Nafion film could have a hierarchical phase-separated structure, as shown in Figure 3. The superstructure, the larger phase-separated structure, is a bicontinuous phase separation between the domain of the main chains (PTFE matrix) and that of the grafted ones, of which the scale is around $150 \AA$. The substructure, the smaller structure, is located in the domain of the graft chains where there is also a bicontinuous phase separation between the region of the hydrophobic blocks and that of the hydrophilic ones (sulfate groups) in the graft chain. The water was selectively absorbed in the hydrophilic region, and the scale is enlarged from $28 \AA$ to $35 \AA$ by the water absorption. However, the decrease in the volume of the hydrophobic region could compensate for the increase in the volume of the hydrophilic region because the hydrophobic block in the graft chain could be more flexible than the main chain. Therefore, the scale of the substructure becomes larger with the increase in water content, whereas that of the superstructure is almost independent of the water content. ${ }^{18}$ 


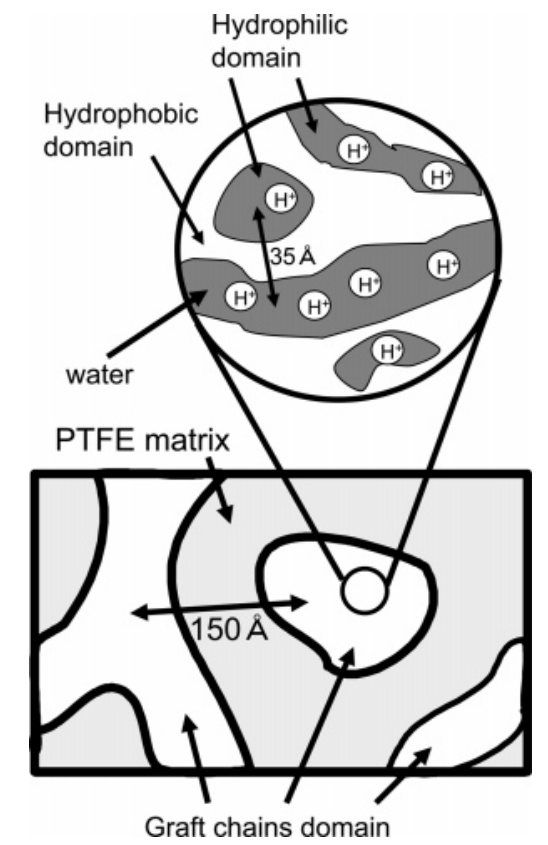

Figure 3. Schematic drawing of the hierarchical structure of Nafion film. In the superstructure, the light gray and white zones represent the domains of PTFE matrix and the aggregates of the graft chains, respectively. In the substructure, the white zone and diagonal line show the regions of hydrophobic and hydrophilic chains in the graft chains, respectively.

4.2. Anomalous Dispersion Effect around the $\mathrm{Cu} K$ Absorption Edge. 4.2.1. Transmission. Figure 4 shows X-ray transmission of the Nafion film with deionized water (sample $\mathrm{B}$ ) and that of the Nafion film absorbing $\mathrm{Cu}$ ions (sample $\mathrm{C}$ ) in the energy range between $8331 \mathrm{eV}$ (Ni K absorption edge) and $8990 \mathrm{eV}$; the $\mathrm{Cu} \mathrm{K}$ absorption edge is $8980 \mathrm{eV}$. The transmission of sample B monotonically increased with the incident X-ray energy due to energy dependence of no anomalous atoms, $\mathrm{H}$, $\mathrm{C}, \mathrm{O}, \mathrm{F}, \mathrm{S}$, and $\mathrm{Cl}$, in this energy range. On the other hand, that of sample $\mathrm{C}$ drastically dropped at the $\mathrm{Cu} \mathrm{K}$ absorption edge. This clearly indicated that sample $\mathrm{C}$ included $\mathrm{Cu}$ atoms.

Here, the density of the $\mathrm{Cu}$ atom, $d_{\mathrm{A}}$, can be found with the transmission data as follows,

$$
d_{\mathrm{A}}=\frac{1}{\left(\sigma_{2}-\sigma_{1}\right) \cdot t} \ln \frac{T_{1}}{T_{2}}
$$

where $T_{1}$ and $T_{2}$ are transmission at the energies across an absorption edge, $\sigma_{1}$ and $\sigma_{2}$ are their absorption coefficients, and $t$ is the thickness of the sample. Following eq 25, the $\mathrm{Cu}$ density, $d_{\mathrm{A}}$, was found to be $7.51 \times 10^{-2} \mathrm{~g} / \mathrm{cm}^{3}$ in sample $\mathrm{C} ; T_{1}=0.679$ (at $E=8980 \mathrm{eV}), T_{2}=0.488($ at $E=8990 \mathrm{eV}), \sigma_{1}=35.8$ $\mathrm{cm}^{2} / \mathrm{g}, \sigma_{2}=287 \mathrm{~cm}^{2} / \mathrm{g}$, and $t=1.75 \times 10^{-2} \mathrm{~cm}$. This means that the weight of $\mathrm{CuCl}_{2}$ corresponded to $8 \%$ in the increase weight ratio, $R=25 \%$, of sample C (see Table 1). Therefore, sample $\mathrm{C}$ absorbed almost the same amount of water as sample $\mathrm{B}(R=19 \%)$. In addition, an area density of $\mathrm{Cu}$ ion $\left(s_{\mathrm{A}}=d_{\mathrm{A}} t\right)$ in sample $C$ was also found to be $2.06 \times 10^{-5} \mathrm{~mol} / \mathrm{cm}^{2}$ from eq 25 , whereas that of the ionic group is $3.27 \times 10^{-5} \mathrm{~mol} / \mathrm{cm}^{2}$ in Nafion $117\left(\mathrm{EW}=1100\right.$ and $\left.s_{\text {Nafion } 117}=3.6 \times 10^{-2} \mathrm{~g} / \mathrm{cm}^{2}\right)$. It was supposed that the charge of the ionic group in sample $\mathrm{C}$ could be screened by the $\mathrm{Cu}$ ion if the $\mathrm{Cu}$ ion is located around the ionic group.

4.2.2. Distribution of $\mathrm{Cu}$ Ion. Figure 5 shows the energy dependence of SAXS profiles of sample $\mathrm{C}$ around the $\mathrm{Cu} \mathrm{K}$ absorption edge. At $E=8990 \mathrm{eV}$, the intensity of the
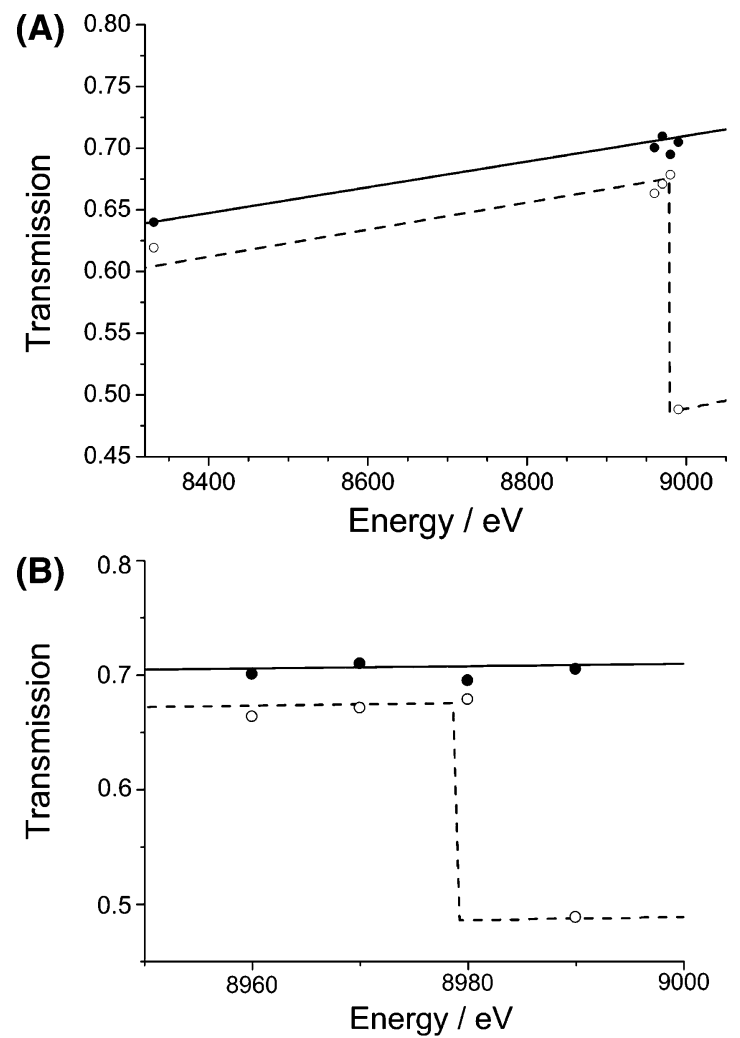

Figure 4. (A) Energy dependence of observed transmission of samples. Closed circles denote the transmission of the Nafion film without $\mathrm{Cu}$ ions (sample B). The solid line also shows the least-squares fitting of those data. Open circles denote the transmission of the Nafion film with $\mathrm{Cu}$ ions (sample $\mathrm{C}$ ). Dashed line shows the convolution of the transmission of $\mathrm{Cu}$ ion, and the solid line: the density of $\mathrm{Cu}$ ion that was calculated with equation 25. (B) The magnified one around the $\mathrm{Cu} \mathrm{K}$ absorption edge.

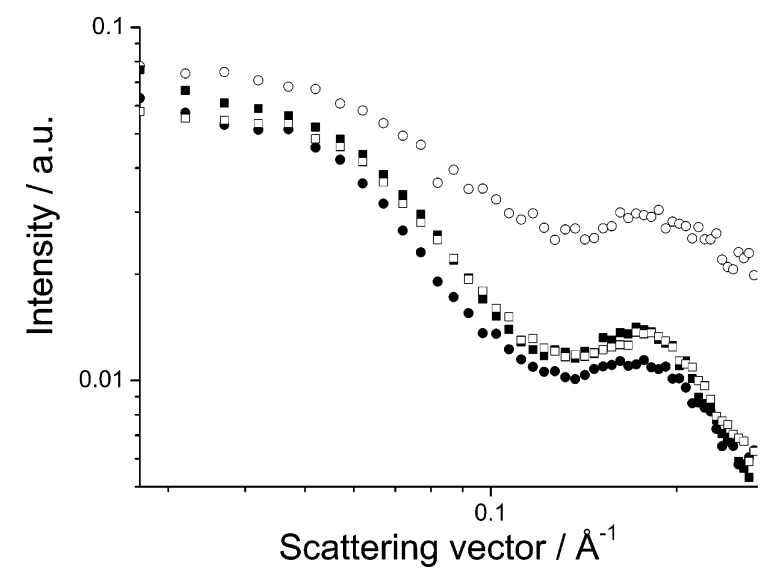

Figure 5. Energy dependence of SAXS profiles of sample $\mathrm{C}$ around the $\mathrm{Cu} \mathrm{K}$ absorption edge. Closed squares, closed circles, open squares, and open circles denote the SAXS profiles at $E=8331 \mathrm{eV}, E=8970$ $\mathrm{eV}, E=8980 \mathrm{eV}$ and $E=8990 \mathrm{eV}$, respectively. At $E=8990 \mathrm{eV}$, the background intensity became stronger due to fluorescence X-ray.

background scattering became stronger due to fluorescence $\mathrm{X}$-ray from the $\mathrm{Cu}$ atoms. This is more clear evidence that sample $\mathrm{C}$ included $\mathrm{Cu}$ ions. However, it is difficult to estimate the amount of fluorescence X-ray in the background scattering. Therefore, we have chosen three SAXS profiles at the energies below the absorption edge, $E_{1}=8331 \mathrm{eV}, E_{2}=8970 \mathrm{eV}$, and $E_{3}=8980 \mathrm{eV}$, to derive the partial structure factor, $G_{\mathrm{AA}}(q)$, corresponding to the distribution of $\mathrm{Cu}$ ions in the Nafion film. For calculation of $G_{\mathrm{AA}}(q)$ following eq 23, the adopted values 
TABLE 2: Anomalous Dispersion Terms of Atomic Form Factors

\begin{tabular}{crc}
\hline energy $[\mathrm{eV}]$ & \multicolumn{1}{c}{$f^{\prime}$} & $f^{\prime}$ \\
\hline 8831 & -2.35 & 0.550 \\
8970 & -6.64 & 0.484 \\
8980 & -10.34 & 0.483
\end{tabular}

of the anomalous dispersion terms in the atomic form factor at these energies are listed in Table 2.

As shown in Figure 6, the partial structure factor of $\mathrm{Cu}$ atoms, $G_{\mathrm{AA}}(q)$, in sample $\mathrm{C}$ was successfully derived from the observed three SAXS intensities. Two clear peaks are found in Figure 6, similar to the standard SAXS profile shown in Figure 2, suggesting the hierarchical distribution of $\mathrm{Cu}$ ions. The position of the second peak at $q=0.18 \AA^{-1}$ is almost the same position as that of the second peak of sample $\mathrm{B}\left(q=0.17 \AA^{-1}\right.$, Figure 2). This clearly indicates that the $\mathrm{Cu}$ ion in the Nafion film is located in the hydrophilic region consisting of water and sulfate groups. Supposing that Nafion film keeps a hierarchical structure after absorbing the $\mathrm{Cu}$ ions and the $\mathrm{Cu}$ ion is located in the region of hydrophilic groups, the distribution of $\mathrm{Cu}$ ions should also be hierarchical. It means that the structure factor of the $\mathrm{Cu}$ ions, $G_{\mathrm{AA}}(q)$, should have the peak corresponding to the superstructure. Therefore, the first peak at $q=0.07 \AA^{-1}$ in $G_{\mathrm{AA}^{-}}$ (q) could be the peak corresponding to this superstructure.

Here, we look back to the result of sample $\mathrm{C}$ by the standard SAXS measurement. When we compared the standard SAXS profile of sample $\mathrm{C}$ with the obtained $G_{\mathrm{AA}}(q)$, we found that the positions of two peaks in $G_{\mathrm{AA}}(q)$ are almost overlapping with those of the standard SAXS profile of sample $C$. This also means that the $\mathrm{Cu}$ ions are located in the hydrophilic region, which distributes hierarchically.

In terms of the relation between the structure and the electric property, the connectivity among the hydrophilic regions becomes higher with the an increase in the water content in Nafion film, and then the electric conductivity also becomes higher, as indicated in Table 1. When Nafion film absorbs $\mathrm{Cu}$ ions, it also absorbs water, and the conectivity among the hydrophilic regions increases. However, the $\mathrm{Cu}$ ion is replaced with a proton as a counterion of a sulfate group, and it creates the degradation of the electric conductivity on the Nafion film, as indicated in Table 1.

\section{Conclusions and Remarks}

We can successfully derive a distribution of $\mathrm{Cu}$ ions in Nafion film from small-angle X-ray scattering intensities by utilizing the anomalous dispersion effect. The $\mathrm{Cu}$ ion is distributed in a region where hydrophilic bases (sulfate bases) gather. This is consistent with the structural model based on the result of standard SAXS experiments. In addition, we can show how to be able to find the density of an anomalous atom in a sample

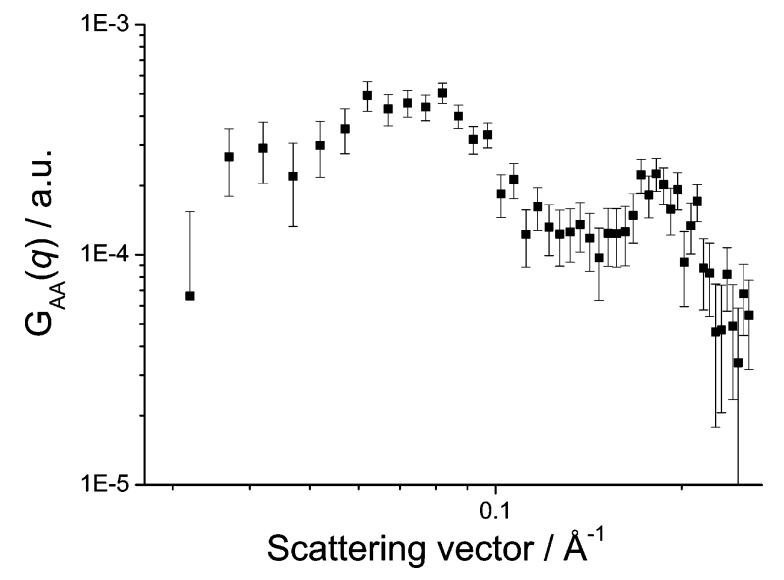

Figure 6. Partial structure factor of $\mathrm{Cu}$ ion $G_{\mathrm{AA}}(q)$ in sample C.

from the difference in transmission below and above the absorption edge, as described in Section 4.2.1. These results indicate that we can obtain various information of a material with an anomalous atom by utilizing the anomalous dispersion effect.

Acknowledgment. The anomalous SAXS experiments were performed under the approval of the Photon Factory Advisory Committee (Proposal No.2004G066).

\section{References and Notes}

(1) Bauen, A.; Hart, D. J. Power Sci. 2000, 86, 482.

(2) Gierke, T. D.; Munn. G. E.; Wilson, F. C. J. Polym. Sci. Polym. Phys. Ed. 1981, 19, 1687.

(3) Fujiyama, M.; Hashimoto, T.; Kawai, H. Macromolecules 1981, 14, 1309.

(4) Rubatat, L.; Gebel, G.; Diat, O. Macromolecules 2004, 37, 7772.

(5) Gebel, G.; Lambard, J. Macromolecules 1997, 30, 7914.

(6) Gebel, G. Polymer 2000, 41, 5829.

(7) Choi, P.; Jalani, N. H.; Datta, R. J. Electrochem. Soc. 2005, 152, E123-E130.

(8) Hendrickson, W. A. Science 1991, 254, 51-58.

(9) Karle, J. Int. J. Quant. Chem. 1980, 7, 357-367.

(10) Fuoss, P. H.; Eisenberger, P.; Warburton, W. K.; Beinnenstock, A. Phys. Rev. Lett. 1981, 46, 1537.

(11) de Robillard, Q.; Guo, X.; Dingenouts, N.; Ballauff, M.; Goerigk, G. Macromol. Symp. 2001, 164, 81 .

(12) Dingenouts, N.; Patel, M.; Rosenfeldt, S.; Pontoni, D.; Narayanan, T.; Ballauff, M. Macromolecules 2004, 37, 8152.

(13) Goerigk, G.; Schweins, R.; Huber, K.; Ballauff, M. Europhys. Lett. 2004, 66 (3), 331 .

(14) Soejima, Y.; Kuwajima, S.; Sugiyama, M.; Annaka, M.; Nakamura, A.; Hiramatsu, N.; Hara, K. J. Phys. Soc. Jpn. 2003, 72, 2110.

(15) Sugiyama, M.; Soejima, Y.; Hara, K.; Takei, F.; Itoh, K.; Mori, K.; Fukunaga, T. Trans. Mater. Res. Soc. Jpn. 2005, 30 (3), 847.

(16) Feigin, L. A.; Svergun, D. I. Structure Analysis by Small-Angle $X$-ray and Neutron Scattering; Plenum Press: New York, 1987.

(17) Sasaki, S. KEK Report 1989, 88-14.

(18) Akai, Y.; Sugiyama, M.; Itoh, K.; Mori, K.; Fukunaga, T. Trans. Mater. Res. Soc. Jpn. 2005, 30 (3) 855. 